\title{
Design and Realization of Digital Pulse Compression in Pulsed Radars Based on Linear Frequency Modulation (LFM) Waveforms Using FPGA

\author{
H. A. Said ${ }^{1}$, A. A. El-Kouny ${ }^{2}$, A. E. El-Henawey ${ }^{1}$ \\ ${ }^{1}$ Faculity of Engineering, Ain Shams University \\ ${ }^{2}$ Misr University of Science and Technology
}

\begin{abstract}
Pulse compression is a method for achieving most of the benefits of a short pulse while keeping within the practical constraints of the peak power limitation. It is usually a suitable substitute for the short pulse waveform except when a long minimum range might be a problem or when maximum immunity to repeater ECM is desired. Pulse compression radars, in addition to overcoming the peak-power limitations, have an EMC (Electromagnetic Compatibility) advantage in that they can be made more tolerant to mutual interference. This is achieved by allowing each pulse-compression radar that operates within a given band to have its own characteristic modulation and its own particular matched filter.. In this paper, we shall implement (LFM) linear frequency modulation digital pulse compression technique using (FPGA) which has distinct advantages compared to other application specific integrated circuits (ASIC) for the purposes of this work. The FPGA provides flexibility, for example, full reconfiguration in milli-seconds and permits a complete single chip solution.
\end{abstract}

Keywords: FPGA, Linear frequency modulation, Digital pulse compression.

\section{Introduction}

Nowadays, Radars are commonly used in Air Traffic Control System. It needs a good presence of target location and good target resolution. Good range resolution can be achieved with a shorter pulse. But on the other hand, shorter pulses need more peak power. The shorter the pulse gets, the more should its peak power be increased so that enough energy is packed into the pulse.

High Peak Power makes the design of transmitters and receivers difficult since the components used to construct these must be able to withstand the peak power. In order to overcome this problem, convert the short pulse into a longer one then use some form of modulation to increase the bandwidth of the long pulse so that the range resolution is not compromised. This used scheme is called the Pulse Compression Technique (PCT) and is used widely in Radar applications where high peak power is undesirable.

Increasing the length of the pulse achieve the reduction in the peak power of it. But, it reduces range resolution. To avoid the compromise in range resolution, some form of encoding must be done within the transmitted pulse, so that it is possible to "compress" a longer pulse into a shorter one in the receiver using suitable signal processing operations.
The easiest form of such encoding is to allow the radar pulse to modulate a waveform or a sequence that is uncorrelated in time but known at the receiver. A crosscorrelation operation at the receiver (using the known transmitted waveform/sequence) will compress the long received waveform/sequence into a short one. This is due to the time auto-correlation properties of the transmitted waveform/sequence, which is max. At zero-lag and almost zero at lags other than zero. The time auto-correlation of a deterministic function $\mathrm{f}(\mathrm{t})$ of time is given by:

$R_{f f}(\tau)=\int_{-\infty}^{\infty} f(t) f^{*}(t+\tau) d t$

And, for a random signal $\mathrm{X}(\mathrm{t})$, it is given by:

$$
R_{X X}(\tau)=E\left[X(t) X^{*}(t+\tau)\right]
$$

The objective of designing a good Pulse Compression system is now to choose an encoding signal that has a very narrow auto-correlation function.

\section{Pulse Compression Radar}

\subsection{LFM Waveform}

It is a frequency modulated waveform in which the carrier frequency varies linearly in time for some specified period (for $\mathrm{CW}$ radar) or within the pulse width (for pulsed radar). To obtain this waveform, the phase must have a quadratic dependence on time. The waveform voltage can be written as

$$
V(t)=V_{0} \cos \phi(t)=V_{0} \cos \left[\omega_{0} t+\theta(t)\right]
$$

Where $\mathrm{V}_{0}$ is the amplitude, $\omega_{0}$ is the carrier frequency, and $\theta(\mathrm{t})$ is the phase.

When

$$
\theta(t)=k t^{2}
$$

The instantaneous frequency:

$$
\omega(t)=\frac{d \phi(t)}{d t}=\omega_{0}+2 k t
$$

Fig. 1 providing intra-pulse modulation. A variant of the linear FM waveform is the linear-step FM waveform, in which the frequency versus time function is not the continuous one. 


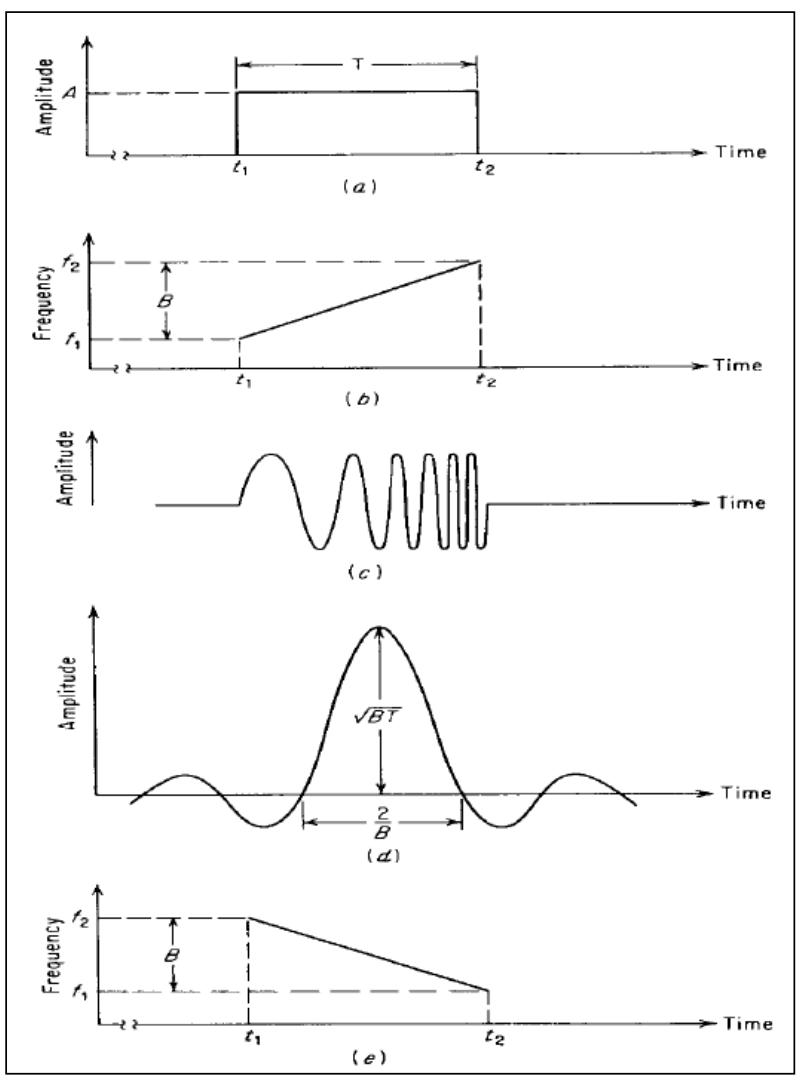

Fig. 1: LFM waveform and its compression:
(a) Transmitted waveform;
(b) Frequency of the transmitted waveform;
(c) Representation of the time waveform;
(d) Output of the pulse-compression filter;
(e) Same as (b) but with decreasing frequency.

\subsection{Matched Filtering and Correlation}

A correlation operation at the radar receiver indicates the presence of a received echo by compressing the received signal in time using its time-correlation properties. Matched Filter performs the correlation operation. In fact, it can be mathematically shown that matched filter using the time reversed complex-conjugate of a signal is equivalent to correlating with the signal. Consider the matched filter operation between a signal $\mathrm{x}(\mathrm{t})$ and an impulse response $\mathrm{h}$ $(\mathrm{t})$ :

$$
y(\tau)=x(t) * h(t)=\int_{-\infty}^{\infty} x(t) h(\tau-t) \cdot d t
$$

Replacing $\mathrm{h}(\mathrm{t})$ by $\mathrm{x}^{*}(-\mathrm{t})$ in equation (6) convert the matched filter operation into an auto correlation operation for signal $x(t)$.

$$
y(\tau)=\int_{-\infty}^{\infty} x(t) x^{*}(\tau-t) \cdot d t=R_{x x}(\tau)
$$

Thus it is obvious that the auto-correlation operation is mathematically equivalent to matched filter with a time-reversed complex conjugate of the signal. In the frequency domain, the product of the Fourier transforms of the signal $x(t)$ and its time-reversed complex-conjugate can represent the matched filter.

$$
R_{x x}(\tau)=F^{-1}\left\{F[x(t)] F\left[x^{*}(-t)\right]\right\}
$$

For the purposes of simulation and digital implementation, the above operation can also be represented using a discrete-time representation. Suppose $x$ (t) is sampled using a sampling duration $T_{s}$ and has a finite number of samples $\mathrm{N}=\mathrm{T} / \mathrm{T}_{\mathrm{s}}$, the auto-correlation sequence can be obtained as:

$$
R_{x x}(m)=\sum_{n=-\infty}^{\infty} x(n) * x^{*}(n-m) \quad \mathrm{m}=0,1, \ldots ., \mathrm{N}-1
$$

Since the auto-correlation sequence is symmetric, it is sufficient to consider only the positive lags. The above operation can be implemented using the matched filter operation. By defining $h(n)=x^{*}(-n)$, we have:

$$
\begin{gathered}
R_{x x}(m)=\sum_{n=-\infty}^{\infty} x(n) h(m-n) \\
R_{x x}(m)=x(n) * x^{*}(-n) \\
R_{x x}(m)=\operatorname{IFFT}\left[\operatorname{FFT}\{x(n)\} \times \operatorname{FFT}\left\{x^{*}(-n)\right\}\right]
\end{gathered}
$$

Where, the FFT and IFFT operations were used to simplify the Correlation operation.

\section{Model of Pulse Compression}

By taking the pulse width is $102.4 \mu \mathrm{s}$, the phase curve is a quadratic curve start from $64 \pi$ to zero at the center of the pulse then goes up again to $64 \pi$ at the end of pulse, and the bandwidth of the chip signal is $2.5 \mathrm{MHz}$.

\subsection{Model of LFM Transmitted Signal}

By applying the previous condition of phase equation we get that:

$$
\varphi(t)=a t^{2}+b t+c
$$

Where

$$
\begin{aligned}
& a=1.220703125 * 10^{10}, \\
& b=-1.25 * 10^{6} \\
& c=64 \pi
\end{aligned}
$$

By finding the equation of frequency we differentiate the phase equation

$$
f(t)=\frac{d}{d t} \varphi(t)=2.44140625 * 10^{10} t-1.25 * 10^{6}
$$

LFM signal (also known as the mathematical expression of the Chirp signal):

$$
s(t)=\operatorname{rect}\left(\frac{t}{T}\right) e^{j 2 \pi\left(f_{c} t+\frac{K}{2} t^{2}\right)}
$$




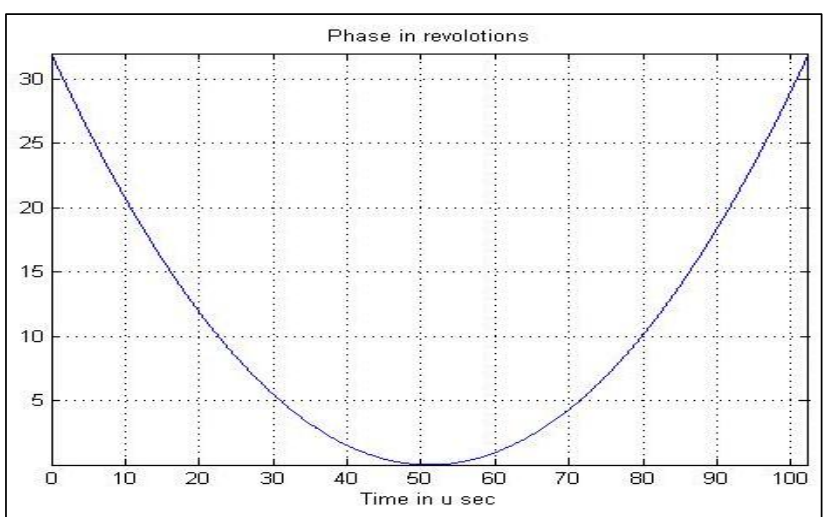

Fig. 2: Time vs. Phase

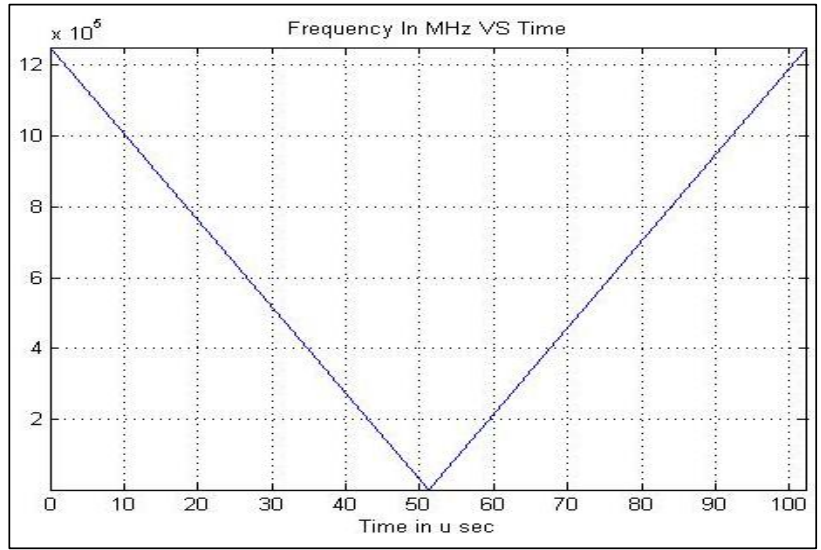

Fig. 3: Time vs. Frequency

Hence, the real and imaginary parts of LFM transmitted signal in time domain will be

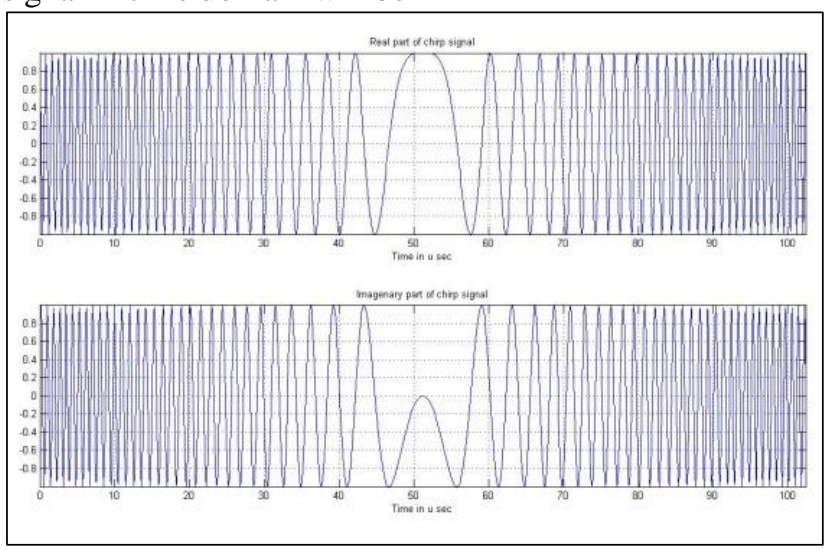

Fig. 4: Real \& Imaginary Parts of LFM Transmitted Signal

\subsection{Model of Matched Filter}

The matched filter impulse response of signal $s(t)$ in time-domain should be as follows:

$$
h(t)=s^{*}\left(t_{0}-t\right)
$$

Where $t_{0}$ is the filter additional delay. Theoretical analysis, can make $t_{0}=0$, so, equation(16) could be rewrite as follows:

$$
h(t)=s^{*}(-t)
$$

By substituting from equation (15) into equation (17) we get:

$$
\begin{gathered}
h(t)=\operatorname{rect}\left(\frac{t}{T}\right) e^{-j \pi K t^{2}} \times e^{j 2 \pi f_{c} t} \\
\stackrel{\mathrm{s}(\mathrm{t})}{\mathrm{h}(\mathrm{t}) \quad \text { So(t) }} \stackrel{\mathrm{S}}{\longrightarrow}
\end{gathered}
$$

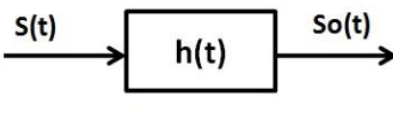

Fig. 5: LFM Signal Matched Filter

As shown in figure 5 , we get the output signal $s_{0}(t)$ from the input signal $s(t)$ after passing through the matched filter $h(t)$

$$
\begin{gathered}
S_{0}(t)=S(t) * h(t) \\
S_{0}(t)=\int_{-\infty}^{\infty} h(u) S(t-u) d u
\end{gathered}
$$

$S_{0}(t)$

$=\int_{-\infty}^{\infty} e^{-j \pi K u^{2}} \operatorname{rect}\left(\frac{u}{T}\right) e^{j 2 \pi f_{c} u}$

$\times e^{j \pi K(t-u)^{2}} \operatorname{rect}\left(\frac{t-u}{T}\right) e^{j 2 \pi f_{c}(t-u)} d u$

\section{When $0 \leq t \leq T$}

$$
\begin{gathered}
S_{0}(t)=\int_{t-T / 2}^{T / 2} e^{j \pi K t^{2}} e^{-j 2 \pi K t u} d u \\
S_{0}(t)=\left.e^{j \pi K t^{2}} \frac{e^{-j 2 \pi K t u}}{-j 2 \pi K t}\right|_{t-T / 2} ^{T / 2} \times e^{j 2 \pi f_{c} t} \\
S_{0}(t)=\frac{\sin \pi K(T-t) t}{\pi K t} e^{j 2 \pi f_{c} t}
\end{gathered}
$$

And when $-T \leq t \leq 0$

$$
S_{0}(t)=\frac{\sin \pi K(T+t) t}{\pi K t} e^{j 2 \pi f_{c} t}
$$

By merge equation (22) and equation (25), we get that:

$$
S_{0}(t)=T \frac{\sin \pi K\left(1-\frac{|t|}{T}\right) t}{\pi K T t} \operatorname{rect}\left(\frac{t}{2 T}\right) e^{j 2 \pi f_{c} t}
$$

Equation (26) is the output impulse response of the LFM matched filter, where the constant carrier frequency is $f_{c}$, and when $t \leq T$ the envelope approximately (sinc) function.

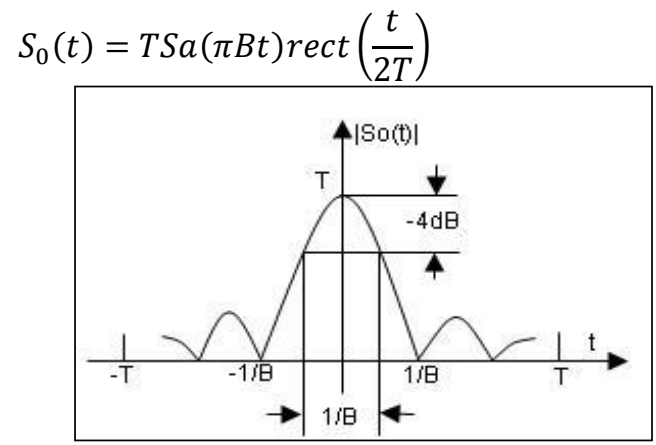

Fig. 6: The Matched Filter Output Signal

The simulation results for the output of the matched filter shown in Figure 7: 


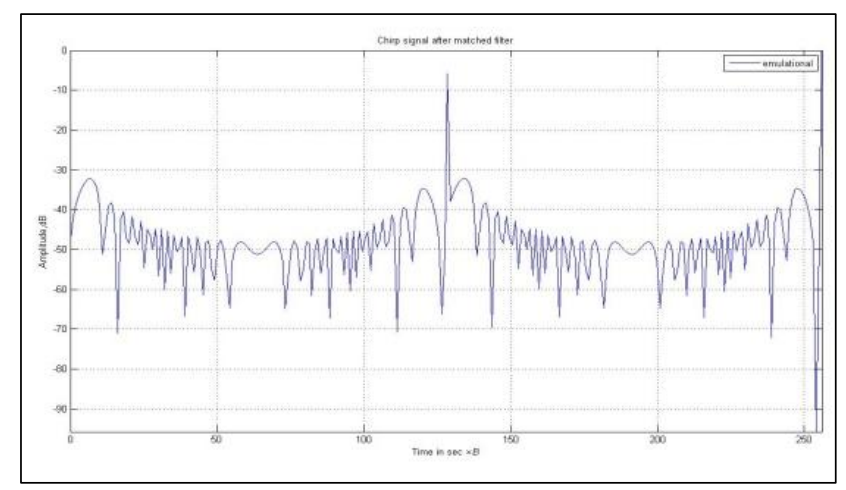

Fig. 7: Chirp Signal after Matched Filtering

\subsection{Targets Simulation Results:}

In this simulation, we can see how the pulse compression affects the range resolution of the radar. Consider the following parameters in (Table 1):

Table 1

Parameters of Model Simulation

\begin{tabular}{lc}
\multicolumn{2}{c}{ Parameters of Model Simulation } \\
\hline Max range & $150 \mathrm{Km}$ \\
Signal waveform & LFM signal \\
Bandwidth & $2.5 \mathrm{MHz}$ \\
Pulse width & $102.4 \mu \mathrm{s}$ \\
Target 1 & $35.5 \mathrm{Km}$ \\
Target 2 & $111 \mathrm{Km}$ \\
Target 3 & $120 \mathrm{Km}$ \\
Target 4 & $125 \mathrm{Km}$ \\
Target 5 & $130 \mathrm{Km}$ \\
\hline \hline
\end{tabular}

The simulation results shown in Figure 8.

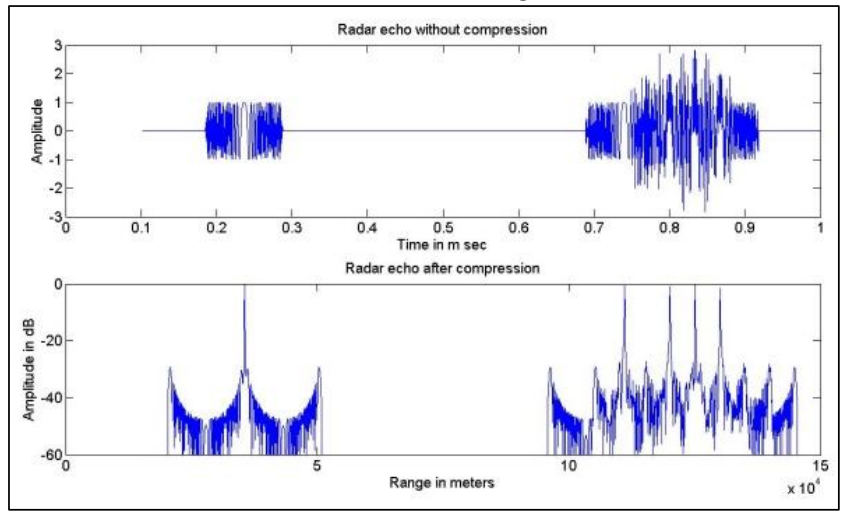

Fig. 8: Simulation Results

\section{Hardware Implementation}

\subsection{Implementation of Transmitted LFM Waveform}

The LFM waveforms are digitally produced when PROMs are addressed by counters initialized by instructions from the radar control process software. The LFM waveforms, consisting of I and Q values, are D/A converted and applied to transmit IF generation circuits. Twelve parallel bits representing the count are output from the counter function and presented to a phase PROM and a timing PROM. The timing PROM is used to determine the start and end of the transmit gate and the waveform generator cutting gate. Waveform generators no. 1 and 2 transmit gates are ORed to provide a complete gate for the duration of the final transmit waveform used by the exciter. The timing PROM provides countdown/up and stop information to the counter. Initially the counter counts down until a zero count is reached. Then the timing PROM changes the counter instruction to count up. An up count continues until the timing PROM sends a stop command.

The phase PROM has been programmed to produce the addresses for the sine/cosine PROMs upon receipt of the counter output. A 4:1 multiplexer selects one of four sine/cosine sources that allow the waveform generator to produce LFM waveforms.

The sine/cosine PROMs contain sine and cosine tables that produce digitally preprogrammed sine and cosine values. The PROM tables contain the sine and cosine values in offset binary code. Every 100 nanoseconds (10$\mathrm{MHz}$ clock cycle), PROM data is read out. This yields a minimum of 8 points per cycle for the highest pulse frequency. The multiplexer selects the counter output for application to the sine/cosine PROMs. This address controls the sine/cosine PROM readout to provide a $\mathrm{CW}$ signal at the frequency selected that is represented by I and $\mathrm{Q}$ output. Fixed maximum or zero outputs may also be selected. The digital LFM waveform outputs, I from the cosine PROM and Q from the sine PROM, are applied to the $\mathrm{D} / \mathrm{A}$ converters. The D/A converters produce the analog LFM cosine and sine (I and Q) waveforms from the digital outputs of the PROMs. Note that the LFM signal starts at maximum frequency, decreases linearly to zero frequency, and then increases linearly to maximum frequency.

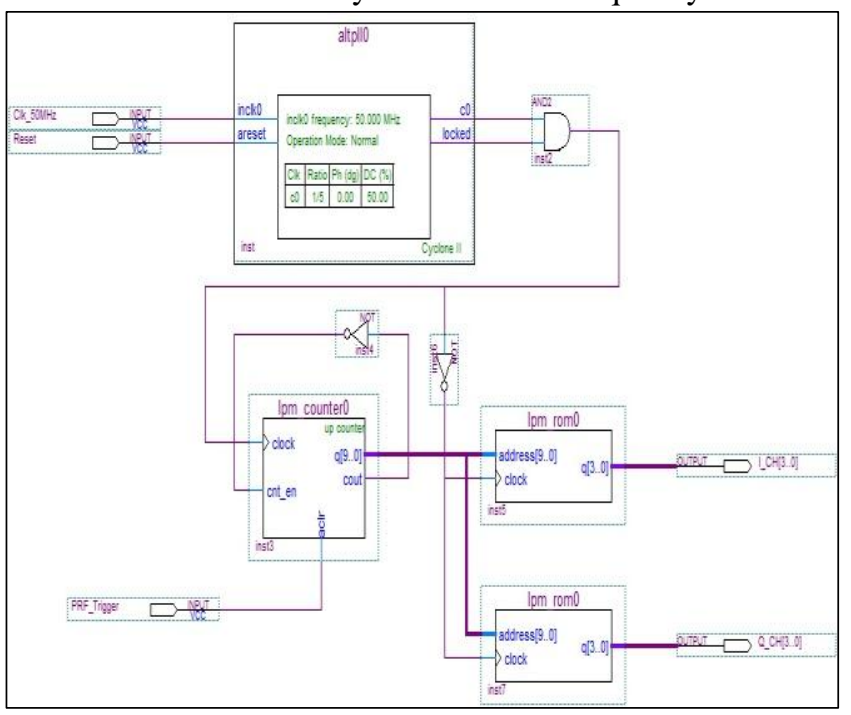

Fig. 9: FPAG Design of Transmitted LFM Waveform

\subsection{Implementation of Matched Filter}

The pulse compressor is the matched filter of the radar receiver. The received signal is correlated to the known transmitted spectrum. Only those target returns that result from transmitter radiation are identified. Interference signals generated externally to the radar are suppressed. 
The pulse-compressor function (Figure 10) is equivalent to the matched filtering provided by a bandpass filter in a conventional radar IF amplifier. A conventional receiver's matched filter extracts amplitude modulation only; phase is irrelevant. A pulse-compression receiver extracts phase information and correlates it to the quadratic phase modulation resulting from the transmitter LFM.

A fast Fourier transform in the pulse-compressor function correlates the received signal return spectrum with the known spectrum of the transmitted signal. The FFT is analogous to a spectrum analyzer.

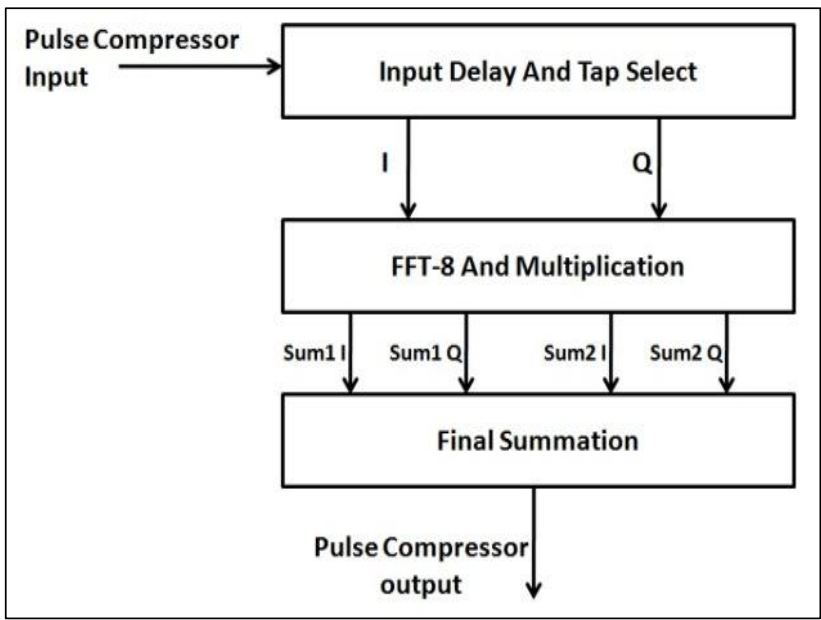

Fig. 10: Pulse Compression Block Diagram

\section{- Implementation of FFT}

A complete FFT-4 is shown in (Figure 11). The FFT matched filter, performing like a spectrum analyzer, samples the input target to determine if all the spectral components are present. These components identify the input waveform as a true target. (Figure 11) shows four groups of phasors similar to the one shown in Figure 3-46. Each group of phasors responds to a separate spectral component. Sum 0 sums the zero phase shift component. Sum 1 sums those spectral components with multiples of $\pi / 2$ (90 degrees) phase rotation between taps; sum 2 sums $\pi$ (180 degrees) multiples; and sum 3 sums $[1-1 / 2 \pi](270$ degrees) multiples. If the output port $\mathrm{A}_{0}, \mathrm{~A}_{1}, \mathrm{~A}_{2}$ and $\mathrm{A}_{3}$ outputs are summed, a maximum response results in correlation. For example, the response of sum $A_{1}$, output port can be shown as:

$$
A_{1}=(0) a_{0}+(\pi / 2) a_{1}+(2 \pi) a_{2}+(3 \pi / 2) a_{3}
$$

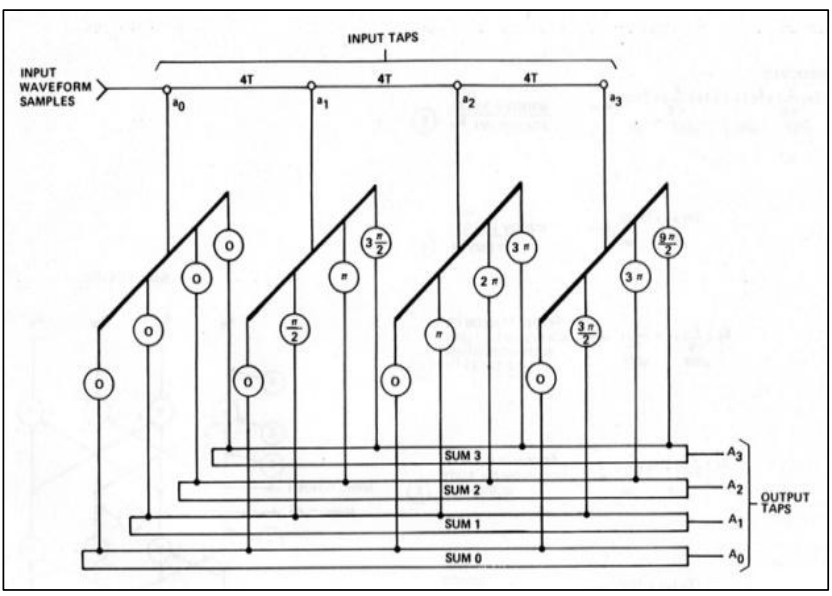

Fig. 11: FFT-4 Phasor Diagram

Although the two basic components of the FFT, the phasors and the summation, have been described as theoretically separate items, (Figure 12) shows the actual FFT-4 implementation. The circles of the diagram represent arithmetic logic units (ALU) which function as simple binary adders and provide the summation quality of the FFT. Each adder has two inputs; each input has either a plus or minus sign indicating the operation (addition or subtraction) performed. If addition is performed, no phase shift results. Subtraction, however, causes a 180 degrees (П) phase shift.

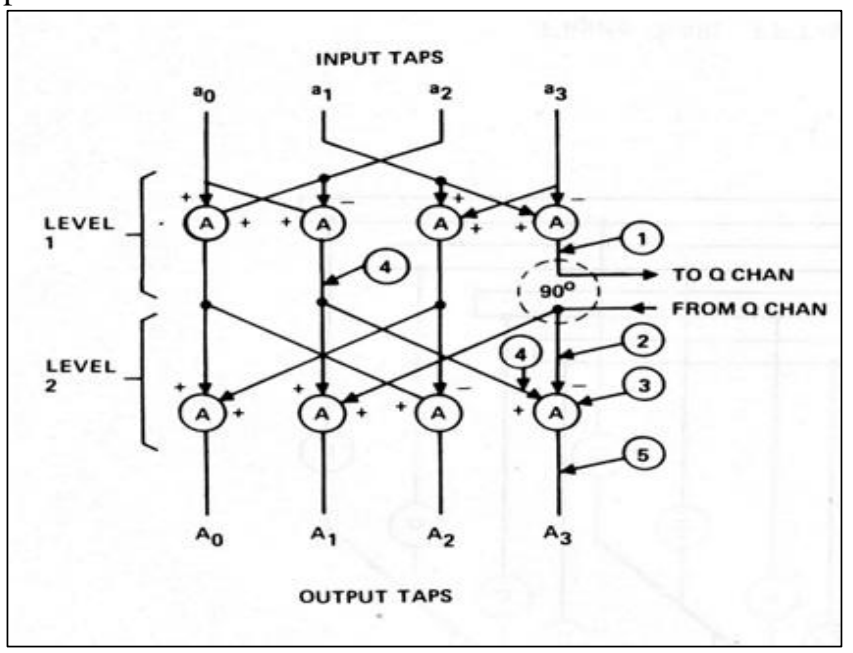

Fig. 12: FFT-4 Adder Implementation

\section{Results}

Experimental results measured from the implemented radar processing model presented in Figure 10. All the synthesized results are single realizations obtained using Altera-Cyclon II FPGA. Using a EP2C8Q208C8N chip and Quartus II as a software design tool, simulation and logic analyzer tool, we get the results as following: 


\subsection{Generation of 4-bit LFM waveform}

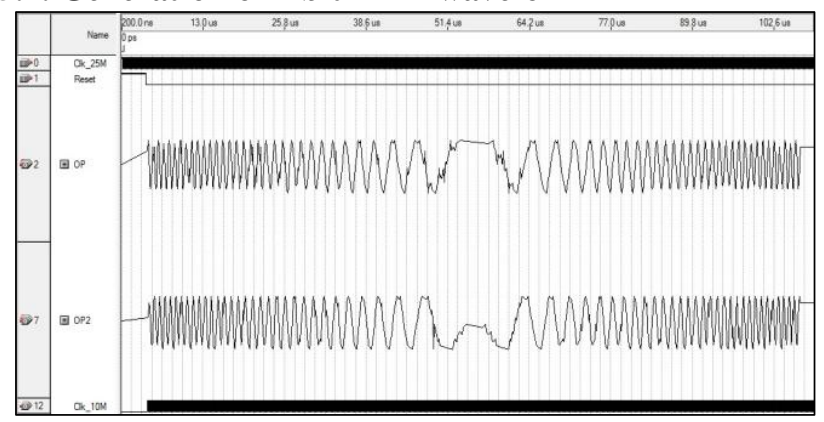

Fig. 12: Generation of I \& Q channels of LFM waveform

\subsection{Resolving of one target:}

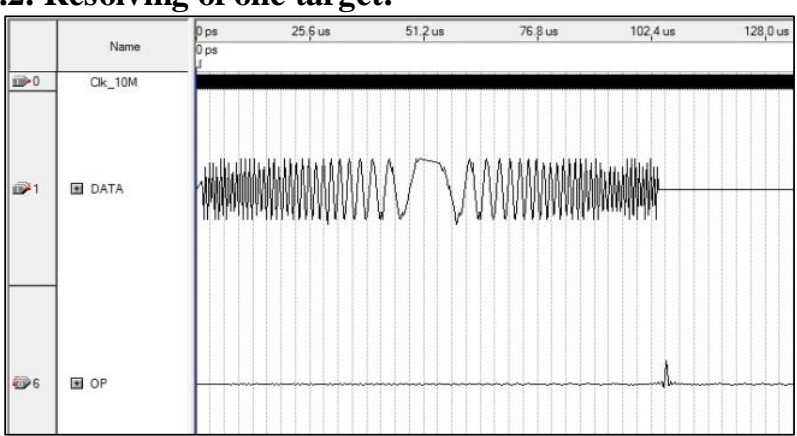

Fig. 11: Time response for matched filter for one target

\section{Conclusion}

A flexible real time implementation of digital pulse compression suitable for all-digital radar receiver architecture. Experimental results obtained presented a very good agreement with the theory. The developed system can further be enhanced to be used for surveillance pulsed radars. The realization based on the FPGA chips makes it easy to change the radar working parameters to adapt the design to different situations which following the concept of software defined radio (SDR).

The proposed architecture is a single chip solution for both generation and receiver processing of pulse compression.

Future research work requires additional investigation in the windowing processing, and also we recommend testing novel windows in frequency domain using FPGA.

\section{References}

[1] FanWang, Huotao Gao, Lin Zhou, Qingchen Zhou, Jie Shi, Yuxiang Sun, " Design and FPGA implementation of digital pulse compression for HF chirp radar based on modified orthogonal transformation", IEICE Electronics Express, Vol.8, P1736-1742, October-25-2011.

[2]Zhisheng Yan, Biyang Wen, Caijun Wang, Chong Zhang, "Design and FPGA implementation of digital pulse compression for chirp based on CPRDIC", IEICE Electronics Express, Vol.6, P 780-786, June10-2009.

[3] J. Lee Blanton,"CUED Medium air-to-air radar using stretch range compression", IEEE 1996 National Radar Conference, Ann Arboe, Michigan, 13-16 May 1996.

[4] Saqib Ejaz, Muhammad Amir Shafiq, Dr. Muhammad Junaid Mughal, "Real time implementation of digital LFM pulse compression technique over acoustic waveguides", International Journal of Engineering \& Technology, Vol.10, No.4.

[5] Enrique Escamilla-Hernandez, Victor Kravchenko, Volodymyr Ponomaryov, Daniel Robles-Camarillo, Luis E. Ramos, "Real time signal compression in radar using FPGA", Cientifica, Vol.12, Num.3, P 131-138, September 2008.

[6] Merril I. Skolnik, "Introduction to radar systems", $3^{\text {rd }}$ edition, McGraw-Hill, 2001.

[7] Merril I. Skolnik, "Radar Handbook", $2^{\text {nd }}$ Edition, McGraw-Hill, 1990.

[8] M. Vamsi Krishna, K. Ravi Kumar, K. Suresh, V. Rejesh, "Radar Pulse compression", International Journal of electronics \& Communication technology, Vol.2, SP-1, December 2011.

[9] Mark-Anthony Govoni, "Linear Frequency Modulation of Stochastic Radar Waveform", Submitted to the Faculty of the Stevens Institute of Technology in partial fulfillment of the requirements for the degree of DOCTOR OF PHILOSOPHY, 2011.

[10] Arojit Roychowdhury, "FIR Filter Design Techniques", M. Tech. credit seminar report, Electronic Systems Group, EE Dept, IIT Bombay, November 2002

[11] V. A. Pogribnoi, T. Leshchinski, I. V. Rozhankovskii, "Methods of enhancing the compression of short LFM signals", Radioelectronics and communications systems, Vol.51, N0.3, P 143-149, 2008.

[12] N. Balaji, M. Srinivasa Rao, K. Subba Rao, S. P. Singh, N. Madhu Sudhana Reddy, "FPGA Implementation of ternary pulse compression sequences", Proceesings of the international multi conference of engineers and computer scientists 2008, Vol.1, 19-21 March 2008 (Hong Kong).

[13] Mark A. Richards, "Time and Frequency Domain Windowing OF LFM Pulses", 29 September 2006.

[14] Wang Peng, Meng Huadong, Wang Xiqin, "Suppressing Autocorrelation Sidelobes of LFM pulse trains with genetic algorithm", Tsinghua Science And Technology, Vol.13, No.6, December 2008.

[15] Bassem R. Mahafza, "Radar Systems Analysis and Design Using MATLAB", chapman \& Hall/CRC, 2000.

[16] Ashok S. Mudukutore, V. Chandrasekar, R. Jeffrey Keeler, "Pulse Compression for Weather Radars", IEEE Transactions on geosciences and remote sensing, Vol.36, No.1, January 1998.

[17] Masanori Shinriki, Hironori Susaki, "Pulse Compression for a Simple Pulse", IEEE Transactions on aerospace and electronic systems, Vol.44, No.4, P.1623-1629, October 2008.

[18] Fun-Bin Duh, Chia-Feng Juang, Chin-Teng Lin, “ Aneural Fuzzy Network Approach to Radar Pulse Compression", IEEE Geoscience and remote sensing letters, Vol.1, No.1, January 2004 